Ecología Austral 28:606-607 Diciembre 2018

Asociación Argentina de Ecología

https://doi.org/10.25260/EA.18.28.3.0.831

\title{
Recordando a Silvia Burkart (07/12/1942-17/01/2018)
}

\author{
Pedro Tognetti, Marina Omacini, Mariano Oyarzabal \& Gustavo Striker
}

Universidad de Buenos Aires, Consejo Nacional de Investigaciones Científicas y Técnicas, Instituto de Investigaciones Fisiológicas y Ecológicas Vinculadas a la Agricultura (IFEVA), Facultad de Agronomía, Buenos Aires, Argentina.

"En aquel Imperio, el Arte de la Cartografía logró tal Perfección que el mapa de una sola Provincia ocupaba toda una Ciudad, y el mapa del Imperio, toda una Provincia. Con el tiempo, estos Mapas Desmesurados no satisficieron y los Colegios de Cartógrafos levantaron un Mapa del Imperio, que tenía el tamaño del Imperio y coincidia puntualmente con él."

\section{"Del Rigor en la Ciencia" Jorge Luis Borges}

Hay por lo menos tres aspectos del epígrafe que nos recuerdan la vida de Silvia Burkart (1942 - 2018). En primer lugar, su capacidad magistral de entender y comunicar de manera sencilla la estructura y el funcionamiento de un paisaje. A diferencia de los Cartógrafos del Imperio, Silvia se esmeraba por hacerlo posible en cualquier escala. En segundo lugar, su rigor, entendido como propiedad y precisión, no se restringía únicamente al ámbito científico. Silvia aplicaba una dedicación especial a la educación que también se trasladaba a su familia y amigos. Tercero, y quizá más evidente, por más que los mapas de Silvia (o de los Cartógrafos) puedan emular con precisión la verdad terrestre, resulta imposible reproducir fielmente la franqueza y la tierna calidez de su sonrisa.

Seguramente muchos lectores de Ecología Austral hayan apreciado y compartido, directa o indirectamente, alguna de las facetas de la vida de Silvia. Pudo haber sido en un aula o en el campo. Alta y sencilla, dictaba con certeza y entusiasmo cursos de grado y posgrado. Quizá leyeron alguno de sus escritos. Por qué no alguno de los detallados relevamientos de la vegetación pampeana y su relación con el paisaje. Tal vez, en un artículo sobre pastizales, reconocieron la figura de Silvia en una foto; muy probablemente Silvia estaría en una posición poco protagónica, oteando gramíneas, haciendo una lista florística o chequeando referencias de una foto aérea de un paisaje. También pudieron haber compartido una reunión de la AsAE, haber charlado con ella acerca de un poster, o haberla visto bailar. Para quienes no tuvieron esa fortuna, éste es un buen momento para indicar también su dedicación para administrar los subsidios, escribir artículos científicos, estar siempre atenta, no sólo al trabajo, sino también a la familia y los amigos. No creemos menos importante destacar que la particular dulzura de una Pavlova era superior si venía de sus manos. Silvia no amaba lo dulce, pero sí el placer de compartirlo.

Silvia era ingeniera agrónoma (Fac Agr. y Vet. Universidad de Buenos Aires, 1966) y Magister Scientiae (Universidad de New South Wales, Australia, 1973). Fue socia de la AsAE desde sus inicios, formó parte en varias ocasiones de la Comisión Directiva y colaboró muy activamente en la organización de la RAE de 1997. Silvia desarrolló toda su carrera como investigadora y docente de la Facultad de Agronomía de la Universidad de Buenos Aires. Se inició como Ayudante-alumna en 1962 y alcanzó el cargo de Profesora Adjunta en 2007. Dictó cursos de grado (Fisiología Vegetal y Fitogeografía, Ecología y Ecología del paisaje), y el curso de posgrado "Análisis de la heterogeneidad de la vegetación", en la Escuela para Graduados Alberto Soriano en la misma facultad.

Sus primeros trabajos se centraron en la fisiología de la germinación de la maleza "chamico" (Datura ferox). Sin embargo, pronto se enfocó en lo que sería su tema central de investigación: el análisis de la fisiografía del paisaje y su relación con la heterogeneidad de la vegetación. Tenía una especial habilidad para detectar patrones sobre fotografías aéreas y proponer hipótesis sobre su relación con la heterogeneidad ambiental en general y de la vegetación en particular. Verificaba luego esas hipótesis mediante censos completos de las especies vegetales y análisis del relieve, la 
hidrografía, el uso de la tierra y los suelos. Su dedicación y gran capacidad para trabajar en grupo la llevaron a colaborar con muchos equipos de científicos y extensionistas en trabajos de relevamiento y cartografía de diferentes tipos de vegetación en casi toda la Argentina, incluyendo los pastizales de la Depresión del Salado, las estepas graminosas de la Patagonia, la sabana del Palmar de Colón, y el parque chaqueño. Aún después de su jubilación en 2007, continuó trabajando con el mismo entusiasmo, dedicación y humildad que siempre la caracterizaron. Poco antes de su fallecimiento se publicó su último trabajo (Perelman et al. 2017, Journal of Vegetation Science), que integra relevamientos realizados durante los últimos 30 años en los pastizales del Rio de la Plata, desde la Pampa Mesopotámica hasta el sur de Pampa Deprimida.

Silvia fue una mujer que supo balancear la dedicación a su familia, a su trabajo en la facultad y en el campo, y a sus amigos, entre los que muy especialmente se contaba su maestro, Rolando León. Fue hija de dos distinguidos botánicos argentinos (Nélida
S. Troncoso y Arturo E. Burkart), esposa de Antonio Hall, y madre de Andrew y Sonia. Quienes la conocimos siempre destacaremos, además de su conocimiento de la flora y la ecología, su generosidad intelectual y su predisposición a escuchar y ayudar a los colegas. Solidaria, humilde y cariñosa, siempre apoyó el crecimiento intelectual y humano de estudiantes y colegas.

Silvia tuvo un vínculo especial con los pastizales y estepas de Argentina, en particular con los de la Pampa Deprimida. Su familia, colegas y amigos dispersaron sus cenizas desde el más que centenario puente de mampostería sobre el Arroyo Buñirigo, cerca de Magdalena, Provincia de Buenos Aires. Ese punto se encuentra a decenas de metros de los censos números 183, 185 y 186, tres entre los más de cuatrocientos censos que ella junto a Rolando León completaron, y que fueron la base del análisis de los pastizales de los partidos de Magdalena y Brandsen. Una de las áreas de vegetación espontánea de la Argentina mejor estudiada desde el punto de vista fitosociológico. Un excelente y perdurable trabajo de una gran persona.

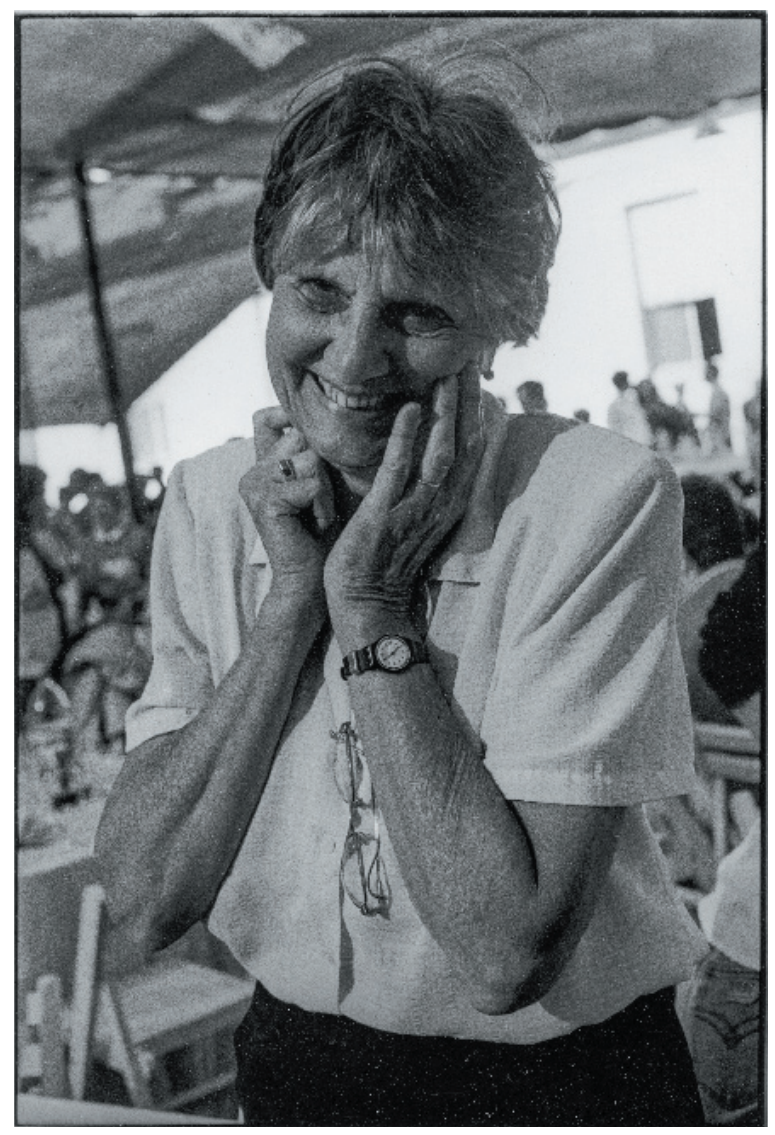

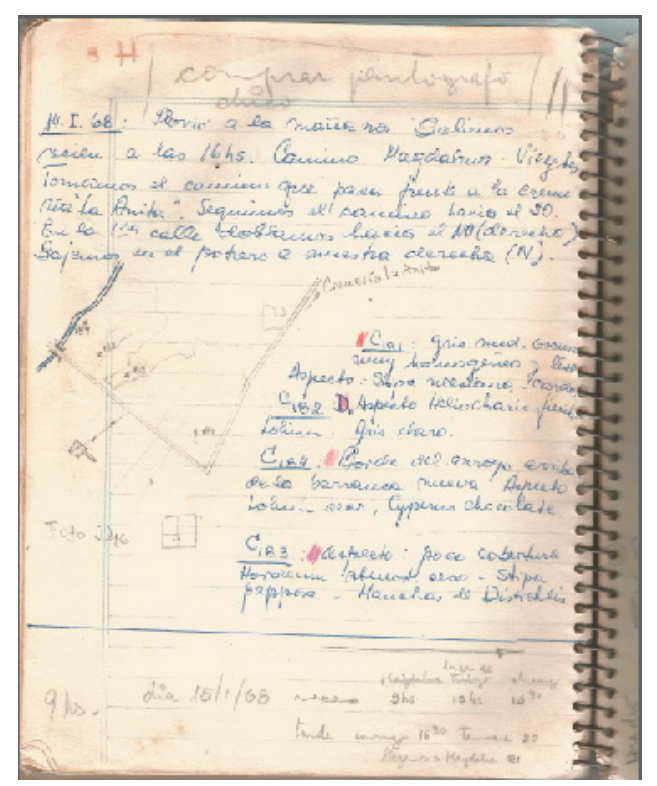

Foto 1. Silvia Burkart (1942 - 2018). Resulta imposible reproducir fielmente la franqueza y la tierna calidez de su sonrisa. Foto María Zorzon (Agronomía Año 100. Universidad de Buenos Aires, 2006)

Foto 2. Cuaderno de Silvia, censos junto al Arroyo Buñirigo, cerca de Magdalena, Provincia de Buenos Aires. 